\title{
Equiluminance: spatial and temporal factors and the contribution of blue-sensitive cones
}

\author{
Patrick Cavanagh \\ Département de Psychologie, Université de Montréal, Montreal, Quebec H3C 3J7, Canada \\ Donald I. A. MacLeod \\ Department of Psychology, University of California at San Diego, La Jolla, California 92093
}

Stuart M. Anstis

Department of Psychology, York University, North York, Ontario M3J 1P3, Canada

Received June 23, 1986; accepted March 11, 1987

\begin{abstract}
Equiluminance ratios for red/green, red/blue and green/blue sine-wave gratings were determined by using a minimum-motion heterochromatic matching technique that permitted reliable settings at temporal frequencies as low as $0.5 \mathrm{~Hz}$. The red/green equiluminance ratio was influenced by temporal but not spatial frequency, the green/blue ratio was influenced by spatial but not temporal frequency, and the red/blue ratio was influenced by both. After bleaching of the blue-sensitive cones, there was no change in equiluminance ratios, indicating no contribution of the blue-sensitive cones to the luminance channel even at low temporal and spatial frequencies. The inhomogeneity of yellow pigmentation within the macular region was identified as the source of the spatialfrequency effect on the blue/green ratio.
\end{abstract}

Several studies have shown that pattern information is divided into chromatic and achromatic pathways very early in the visual system. ${ }^{1-4}$ Opponency between cone responses contributes to the chromatic pathway, whereas summation of cone responses contributes to the nonopponent, or luminance, pathway. Our paper is concerned with the relative contributions of the different cone mechanisms to the luminance channel at different temporal and spatial frequencies and, in particular, with whether the short-wavelength (B) cones contribute at all.

To evaluate the relative contributions to the luminance channel, we determined the null or equiluminance point at which two colors contribute equally. Our stimulus, which will be described shortly, is constructed so that motion is present only if there is a luminance difference between the two colors being evaluated. We also varied the spatial and temporal frequencies used in presenting the stimulus. Cells at the retinal ganglion level can show a range of preferences for spatial and temporal frequency. ${ }^{5}$ For example, the nonopponent cells respond best to low spatial and high temporal frequencies, whereas the color-opponent cells prefer high spatial and low temporal frequencies. ${ }^{1-3}$ These two cell populations project to separate layers of the lateral geniculate (magnocellular and parvocellular, respectively). ${ }^{6}$ It has been argued ${ }^{7}$ that both are involved in carrying achromatic information; the magnocellular layer is principally nonopponent, and the parvocellular layer, although carrying color-opponent information for low spatial and low temporal frequencies, carries nonopponent information at high spatial and temporal frequencies. This separation of achromatic information into two distinct pathways at an early level calls into question the notion of a monolithic luminance pathway. If there are functionally distinct luminance pathways with different spatiotemporal properties, we may be able to identify them by changes in the relative contributions of the various cone mechanisms, and therefore changes in equiluminance settings, as a function of spatial and temporal frequency.

The contribution of the B cones to luminance is currently contested. Results of physiological studies suggest that these cones have very little input to spectrally nonopponent retinal ganglion cells. ${ }^{1-4}$ Eisner and MacLeod ${ }^{8}$ argued on the basis of heterochromatic flicker measurements that $B$ cones made no contribution to luminance. Tansley and Boynton ${ }^{9}$ made the same argument based on minimally distinct border measurements. A weakness in these studies is that they rely directly or indirectly on relatively high spatial or temporal frequencies to evaluate luminance, and there is evidence ${ }^{10-12}$ that the sensitivity of $B$ cones or their associated pathways falls rapidly as spatial or temporal frequency increases. This makes it desirable to test for a B-cone contribution by using the lowest possible spatial and temporal frequencies.

Drum $^{13}$ showed that when white and yellow stimuli were equated for flicker thresholds, the detection threshold for the white stimulus tended to be slightly lower than that for the yellow stimulus. He suggested that his result could be explained most plausibly by a B-cone contribution to the luminance pathway for the low spatial and temporal frequencies involved in detection but not for the high temporal frequency involved in flicker. Involvement of chromatic channels in detection is an unlikely alternative explanation because it was the achromatic, white stimulus that had the lower threshold. 
In this paper we examine these issues by determining whether equiluminance settings vary with the spatial and temporal frequency of the stimulus. We modified the minimum-motion heterochromatic matching technique ${ }^{14,15}$ for use with sinusoidally modulated gratings. This technique produces results like those of conventional flicker photometry, but it has the advantage of permitting very precise matches at either high or low temporal and spatial frequencies.

The results that we present indicate greater short-wavelength sensitivity at low spatial frequencies, suggesting that $\mathrm{B}$ cones do contribute to achromatic sensitivity, since the signal from these cones is much attenuated at high frequencies. But when we repeat the experiment after selectively bleaching the $\mathrm{B}$ cones to suppress their contribution, the matches at all spatial frequencies remain unchanged. From this and other experiments we conclude that the spatialfrequency dependence of heterochromatic matches comes about because of inhomogeneities in macular pigmentation and not because the $B$ cones affect the matches.

\section{THE MINIMUM-MOTION STIMULUS}

The minimum-motion stimulus is relatively complex and needs to be discussed in detail. We will first describe the simpler, four-stroke cycle stimulus that we have used in previous studies ${ }^{14,15}$ and then describe how we have adapted this technique to work with spatially and temporally sinusoidal stimuli.

The original stimulus involved the alternation of red/ green gratings with luminance gratings. A colored squarewave grating of red and green stripes is briefly presented and then replaced by an overlapping grating of light and dark yellow stripes displaced by half a bar width to the right (Fig. 1). Apparent motion is seen in a direction that depends on the relative luminances of the red and green stripes. If the red stripes are darker than the green, the red stripes are seen as jumping to the left into the succeeding dark yellow stripes (Fig. 1a). If the red stripes are lighter than the green, they appear to jump to the right, into the succeeding light stripes (Fig. 1b). If the red and green stripes are equally bright, then no motion is seen. Adding two more gratings produces a continuous four-stroke cycle. Frames 1 and 3 are gratings of red and green stripes, and frames 2 and 4 are gratings of light and dark yellow stripes. The cycle repeats endlessly, giving the impression of continuous apparent motion.

It is straightforward to change the spatial stimuli from square waves to sine waves. The appropriate substitution for the temporal modulation can be understood by referring to Fig. 1. Notice that the two color gratings, T1 and T3, are simply contrast-reversed copies of each other. We can consider these two gratings as one grating undergoing a periodic contrast reversal. In fact, the grating passes through three contrast levels, positive contrast, zero contrast, negative contrast, and zero contrast again, in the four stages, T1-T4, of the cycle [Fig. 1(c)]. The two luminance gratings, T2 and $\mathrm{T} 4$, can also be described as a single luminance grating undergoing a similar contrast reversal over time. Note that the color grating reaches its maximum contrast when the luminance grating is at zero contrast; that is, the waveforms of the two gratings are similar but they are offset by onequarter cycle in time. We can therefore replace these two
T1

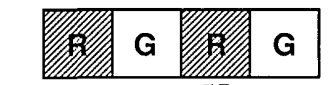

(a) $\bigsqcup^{\text {(b) }}$

T2

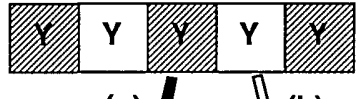

(a) $\quad \zeta(b)$

T3

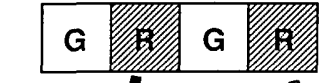

T4
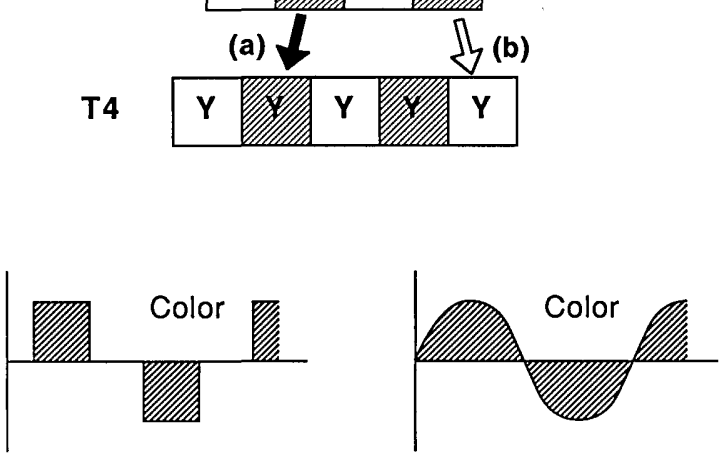

(c)

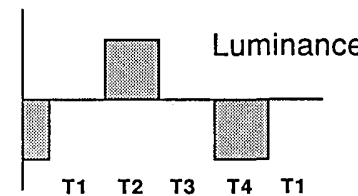

(d)

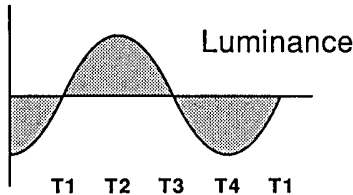

Fig. 1. Four colored gratings are exposed in a repetitive sequence, at times T1-T4, on the screen of a computer-controlled television. Positions of the gratings were superimposed, not displaced vertically as illustrated. Each grating was displaced sideways by onequarter cycle (half a bar width) from its predecessor. The direction of the apparent motion, shown by the arrows, depended on the luminance. (a) When the red bars were darker than the green bars, the red bars in the grating at time T1 (or T3) appeared to jump leftward to the dark yellow bars in the grating at time T2 (or T4) (b) Conversely, when the red bars were lighter than the green bars, the red bars appeared to jump rightward to the light yellow bars. The luminance contrast of the light and dark yellow bars was fixed, typically at $10 \%$. The luminance contrast of the red and green bars was initially unknown but under the control of the observer. (c) The temporal waveforms of the color and luminance gratings in the four-stroke cycle. (d) Equivalent sinusoidal waveforms for the color and luminance gratings.

square-wave temporal waveforms with sinusoidal modulation in time of the two gratings with their modulations offset by $90^{\circ}$ of temporal phase. The following mathematical analysis reveals the motion inherent in this stimulus as well as the criteria for optimizing its presentation.

Our stimulus is produced by superimposing two gratings, both in counterphase flicker with the same spatial and temporal frequencies. Neither of these gratings alone has any net energy moving leftward or rightward; it is only the interaction of the two gratings that can produce a motion percept. Each of the spatiotemporal gratings that we use is the product of sinusoidal modulation in space and time:

$$
\sin \left(2 \pi f_{S} x\right) \sin \left(2 \pi f_{T} t\right),
$$

where $f_{S}, f_{T}$ are the spatial and temporal frequencies, respectively. The two gratings are $90^{\circ}$ out of phase spatially (i.e., at sine and cosine spatial phases), and their temporal variations are also $90^{\circ}$ out of phase (i.e., at sine and cosine temporal phases). If the two gratings were luminance gratings of 
the same amplitude, their sum would be a single, smoothly moving sine-wave grating, with the direction being leftward if the sign of the cosine grating were positive or rightward if it were negative. This is shown by the identity

$$
\begin{aligned}
\sin \left(2 \pi f_{S} x\right) \sin \left(2 \pi f_{T} t\right)+\cos \left(2 \pi f_{S} x\right) \cos \left(2 \pi f_{T} t\right) & \\
& =\cos \left[2 \pi\left(f_{S} x+f_{T} t\right)\right],
\end{aligned}
$$

which defines the leftward motion of a sine wave. Inverting the cosine terms (a $180^{\circ}$ phase change) produces rightward motion:

$$
\begin{aligned}
\sin \left(2 \pi f_{S} x\right) \sin \left(2 \pi f_{T} t\right)-\cos \left(2 \pi f_{S} x\right) \cos \left(2 \pi f_{T} t\right) & \\
& =\cos \left[2 \pi\left(f_{S} x-f_{T} t\right)\right] .
\end{aligned}
$$

This technique of converting a reversal in contrast into a reversal of motion is the same technique that we used in the simpler four-stroke cycle stimulus of Fig. 1, but now we are using sinusoidal stimuli in space and time. Our stimulus is made up of one luminance grating and one color grating, not two luminance gratings. The second luminance grating is present only when there is a luminance mismatch between the two colors in the color grating.

In our stimulus, the first of the gratings, which we will label the color grating, varies in chrominance and has a luminance variation determined by the observer's setting, while the second grating varies only in luminance. Once the observer has nulled the luminance component in the color grating, the stimulus can no longer generate motion, only flicker, and the equiluminance point has been determined. The color grating is produced by summing, for example, red and green spatiotemporal sine-wave gratings $180^{\circ}$ out of phase (see Fig. 2). If the two sine waves have identical luminance amplitudes, they will have the same sum everywhere and so vary in chrominance but not in luminance (Fig. $2 b$ ). If their two amplitudes are not equal, there will be a sinusoidal luminance variation whose amplitude will be the difference between the red and green luminance amplitudes. This luminance variation will have the same phase as the red component of the color grating if this component has the greater amplitude [Fig. 2(a)] and the same phase as the green component (a sign reversal or, equivalently, a shift of $180^{\circ}$ ) if that component has the greater amplitude [Fig. 2(c)].

Combined with the color grating is a luminance grating that differs from it by $90^{\circ}$ in both spatial and temporal phases and that varies only in luminance. Since the luminance grating has no chromatic variation that can interact with that of the color grating, the result of combining this grating with the first depends solely on the sign of the luminance component of the color grating. The final result, as shown in the right-hand column of Fig. 2, will be motion to the right if the red amplitude is greater than the green [Fig. 2(a)]; no motion, just flicker, when red and green amplitudes are equal [Fig. 2(b)]; or motion to the left if the green amplitude is greater than the red [Fig. 2(c)]. The observer's task is therefore to find the point at which motion reverses from leftward to rightward.

Our stimulus can thus be represented as

$$
\begin{aligned}
R(x, t)= & 0.5 L_{R}\left\{\left[1+m \sin \left(2 \pi f_{S} x\right) \sin \left(2 \pi f_{T} t\right)\right]\right. \\
& \left.+\left[1+\cos \left(2 \pi f_{S} x\right) \cos \left(2 \pi f_{T} t\right)\right]\right\}, \\
G(x, t)= & 0.5 L_{G}\left\{\left[1+m \sin \left(2 \pi f_{S} x\right) \sin \left(2 \pi f_{T} t\right)\right]\right. \\
& \left.+\left[1-\cos \left(2 \pi f_{S} x\right) \cos \left(2 \pi f_{T} t\right)\right]\right\},
\end{aligned}
$$

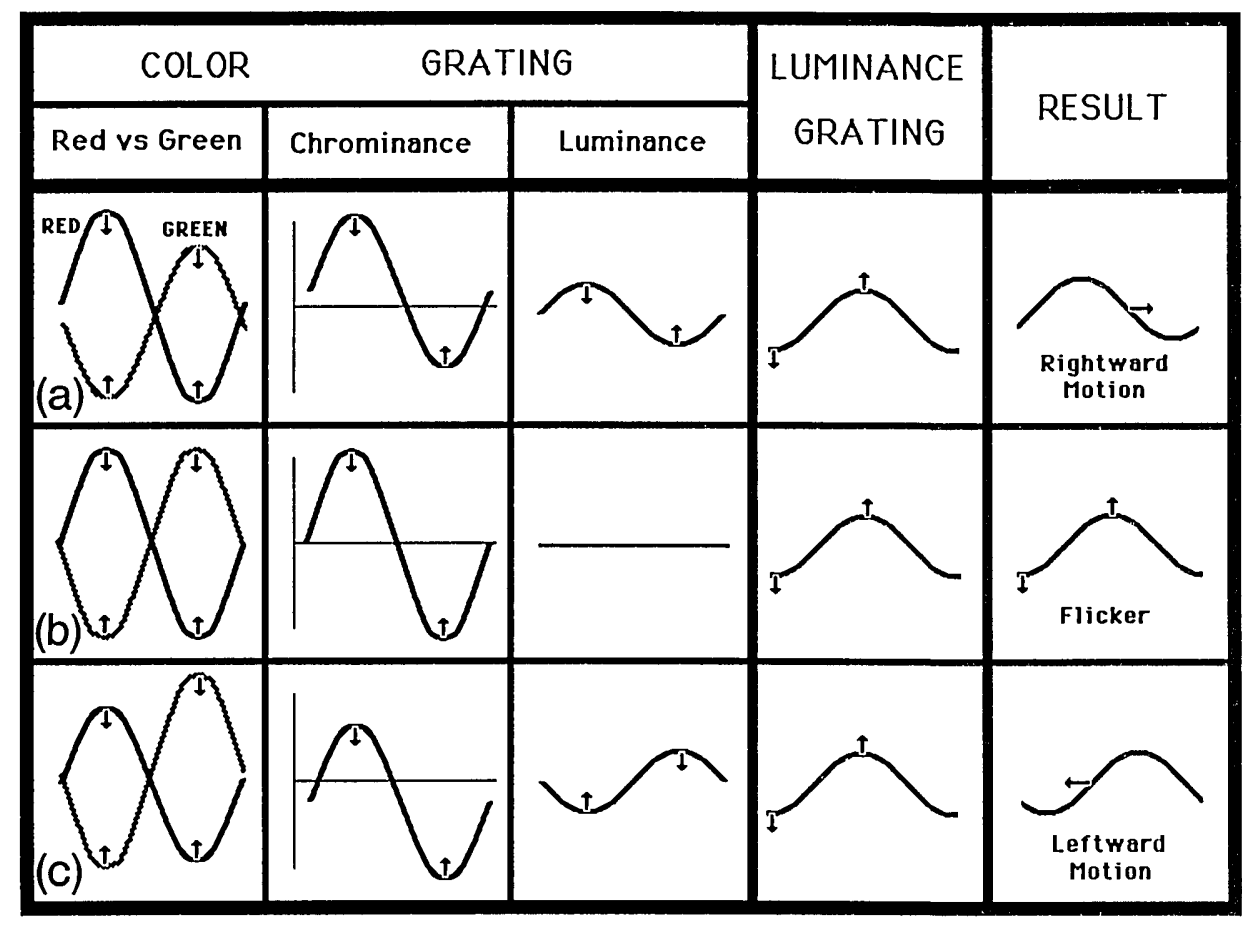

Fig. 2. The superposition of a counterphasing color grating and a counterphasing luminance grating differing by $90^{\circ}$ in spatial and temporal phase is shown decomposed into its various components. In (a) the red variation has a greater amplitude than the green, in (b) it has an equal amplitude, and in (c) it has a smaller amplitude. The color grating is shown as the individual red and green waveforms and then decomposed into separate chrominance and luminance waveforms. The individual red and green waveforms have $100 \%$ contrast: their minimum value is 0 $\mathrm{cd} / \mathrm{m}^{2}$. The horizontal scale in all panels is space, and the arrows indicate changes over time. The final column depicts the resulting motion stimulus. 


\section{m, MODULATION OF} LUMINANCE GRATING

(a) $\mathrm{HIGH}$
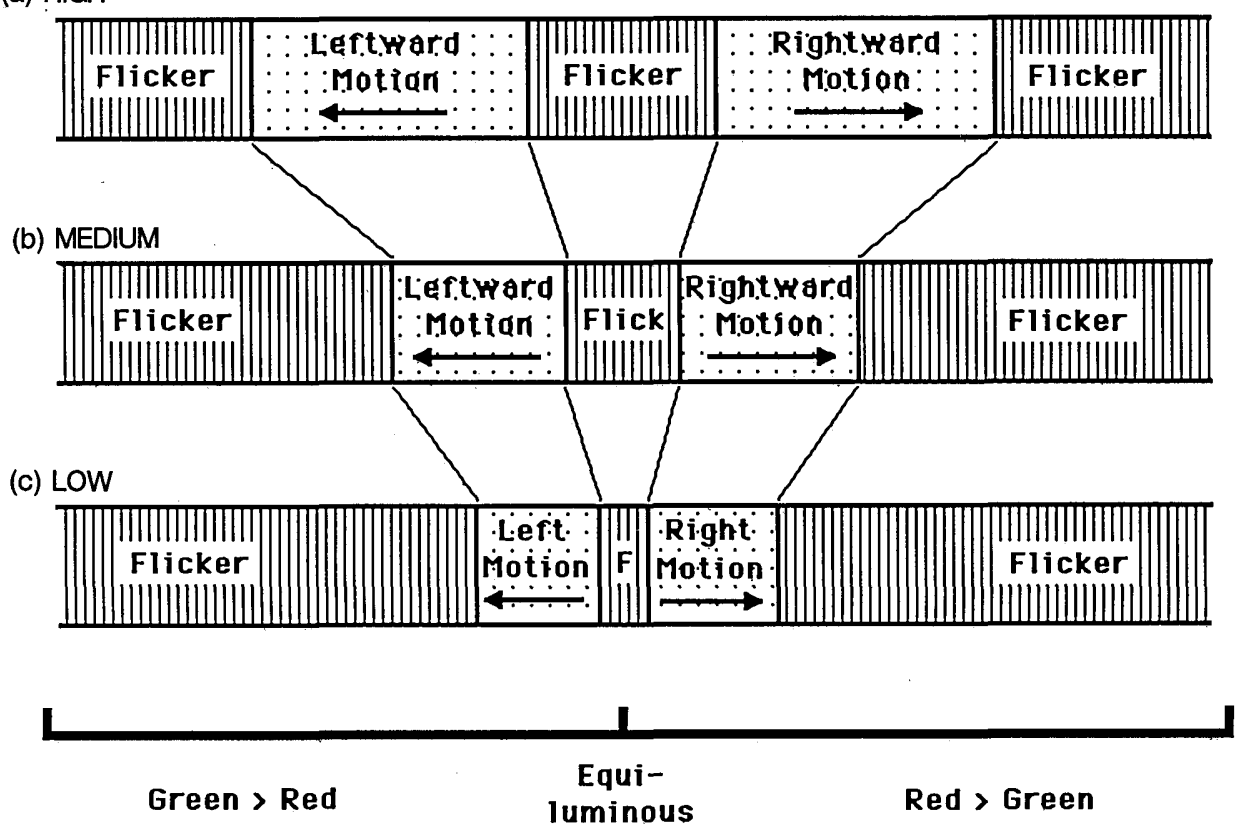

\section{Red vs. Green Amplitude in Color Grating \\ $\left(L_{R}-L_{G}\right)$}

Fig. 3. The range of visible motion in the stimulus as a function of the luminance mismatch between the two colors (red and green here) depends on whether the amplitude of the luminance grating, $m$, is (a) high, (b) medium, or (c) low. When its amplitude is large [say, $15 \%$ as in (a)], motion can be seen over a large range surrounding equiluminance, thus aiding the observer make the setting if the initial value is far removed from equiluminance; however, there is, as well, a large, central range of ambiguous or no motion that makes the exact setting difficult. When its amplitude is small [say, $5 \%$ as in (c)], the exact equiluminance point is more easily located; however, it is difficult to know the appropriate direction of adjustments to make to approach equiluminance if the initial value falls in the large flanking flicker areas.

where $R(x, t)$ and $G(x, t)$ are the luminances of the red and green phosphors; $L_{R}$ and $L_{G}$ are their average luminances, one of which the observer adjusts to achieve a motion null; and $m$ is the contrast of the luminance grating. The sine terms represent the yellow grating (both red and green terms have the same phase), and the cosine terms represent the $\mathrm{red} /$ green grating (red and green terms are $180^{\circ}$ out of phase). Notice that changing $L_{R}$ or $L_{G}$ affects the red/green luminance ratio equally in the color grating and the yellow grating. As a result, the space-averaged chromaticities of the two gratings remain matched at all settings. The spatiotemporal variation of the total luminance is obtained by summing the contributions of the two phosphors in Eqs. (1) and (2). Rewriting in terms of two moving components, one rightward and one leftward, we obtain

$$
\begin{aligned}
L(x, t)= & R(x, t)+G(x, t) \\
= & L_{R}+L_{G}+0.5\left[m\left(L_{R}+L_{G}\right)\right. \\
& \left.+L_{R}-L_{G}\right] \cos \left[2 \pi\left(f_{S} x-f_{T} t\right)\right]+0.5\left[m\left(L_{R}+L_{G}\right)\right. \\
& \left.+L_{G}-L_{R}\right] \cos \left[2 \pi\left(f_{S} x+f_{T} t\right)\right] .
\end{aligned}
$$

From this it can be seen that when $L_{R}>L_{G}$ the rightward motion component (the first cosine) has the higher contrast, and vice versa. When two oppositely moving components are summed, motion is seen in the direction of the component of the higher contrast. ${ }^{16,17}$ When the red and green luminances are equal, the contrasts of the two components are equal and no motion, only flicker, is seen. The flicker has two components, a low-contrast luminance flicker of amplitude $m$ and a large-amplitude chromatic flicker. The motion produced by slight deviations from equiluminance must be detected in the presence of these two masking components. Stromeyer et al. ${ }^{17}$ have shown that the presence of a counterphase mask of moderate contrast may facilitate the detection of motion.

The difference between the leftward and rightward contrasts reaches a maximum when the luminance difference between the two colors approximately equals the amplitude of the luminance grating. For example, when

$$
L_{R}-L_{G}=m\left(L_{R}+L_{G}\right),
$$

substitution for $L_{R}-L_{G}$ in Eq. (3) reveals that the leftward grating (the second cosine) has zero contrast, and thus only rightward motion is present. For much greater luminance mismatches, the contrasts of the leftward and rightward motion components are nearly equal, and the predominant impression is of flicker rather than of motion (see Fig. 3).

Two additional considerations are important in constructing the stimulus: the amplitude and the color of the luminance grating.

As described above, the combination of the luminance grating with the luminance component of the color grating 
produces motion and no flicker when the amplitude of the luminance grating and that of the luminance component of the color grating are identical [i.e., $L_{R}-L_{G}=m\left(L_{R}+L_{G}\right)$ or $L_{G}-L_{R}=m\left(L_{R}+L_{G}\right)$ in Eqs. (1)-(3)]. Any difference in their amplitudes results in a moving grating whose amplitude is equal to the smaller of the two and a flickering grating whose amplitude is equal to their difference. There are two situations in which the flickering grating may have sufficient amplitude to interfere with the detection of the motion $^{17}$ (see Fig. 3). If the amplitude of the luminance component of the color grating is small while that of the luminance grating is large, the resulting flicker can mask the motion that indicates small deviations from equiluminance (the central flicker region in Fig. 3). Conversely, if the luminance component of the color grating is large (that is, if there is a significant mismatch in the red and green luminances) while the amplitude of the luminance grating is small, flicker may again mask the motion (the outer flicker regions in Fig. 3). The observer may confuse this flicker with the flicker that is seen at equiluminance, and adjustments to correct the setting may be difficult to make. To permit the most sensitive detection of the motion produced by combining the two gratings, the amplitude of the luminance grating should be relatively small, but not so small that the range within which motion can be seen is too narrow. We found that $10 \%$ contrast for the luminance grating was optimal.

The choice of the chromaticity coordinates of the luminance grating can affect the visibility of the motion if the chosen coordinates differ from the average chromaticity of the color grating and so produce significant color flicker. We therefore arranged conditions, as specified in Eqs. (1) and (2), such that the luminance grating always had the same chromaticity coordinates and the same mean luminance as the color grating. Since the color gratings were constructed by superimposing two differently colored sine waves $180^{\circ}$ out of phase, the luminance gratings were constructed by superimposing sine-wave gratings of the same two colors and mean luminances as before but now in phase and of lower amplitude. Whenever the observer adjusted the relative amplitudes, $L_{R}$ and $L_{G}$, of the two colors in the color grating, the relative amplitudes were also adjusted in the same proportions, $m L_{R}$ and $m L_{G}$, in the luminance grating. The luminance grating was yellow when the color grating was red/green, magenta when the color grating was red/ blue, and cyan when the color grating was blue/green.

In the first experiment, we used the minimum-motion technique to find the equiluminance ratio for red/green, red/ blue, and green/blue sine-wave gratings over a range of spatial and temporal frequencies extending down to $0.5 \mathrm{~Hz}$ and 0.5 cycle per degree (cpd). Although these stimuli did not stimulate the individual cone mechanisms in isolation, any difference in the spatial or temporal characteristics of the cone mechanisms should be reflected indirectly in our results. In the second experiment, we directly assessed the contribution of the $\mathrm{B}$ cones by bleaching them and repeating our measurements.

We were interested in the effects of spatial and temporal frequency on equiluminance for practical reasons as well. In order to study the opponent color pathways in isolation, it is essential to construct stimuli that provide no pattern information to the luminance pathway. If equiluminance is not influenced by either spatial or temporal frequency, then a single adjustment of relative contrast between the colors involved would be sufficient to ensure equiluminance for arbitrarily complex spatiotemporal stimuli. If, however, spatial and/or temporal frequency influences equiluminance settings, then significant constraints are placed on the types of chromatic images that can be used.

\section{EXPERIMENT 1. SPATIAL AND TEMPORAL FREQUENCY}

\section{Procedure}

The display was presented on a 19-in. ( 48-cm) Conrac 5411 RGB monitor controlled by a Grinnell 270 color graphics system having $512 \times 480$ pixel spatial resolution, 256 intensity levels per color, and a $30-\mathrm{Hz}$ interlaced raster. The phosphors of the monitor were determined by spectroradiometry to have Commission International de L'Eclairage (CIE) $x$ and $y$ coordinates of 0.596 and 0.346 for red, 0.293 and 0.604 for green, and 0.149 and 0.069 for blue. Internal look-up tables in the Grinnell were used to linearize the luminance output of each of the phosphors independently. The stimuli covered $27 \mathrm{~cm} \times 27 \mathrm{~cm}$ on the screen and were viewed from a distance of $7.73 \mathrm{~m}$ subtending a visual angle of $2^{\circ}$. Each observer viewed the display through a five-element achromatizing lens, which corrected longitudinal chromatic aberration without introducing transverse magnification errors ${ }^{18}$ and had a 2-mm aperture, while his or her head was fixed with a dental-impression bite bar. There was a centralfixation bull's-eye $15^{\prime}$ in diameter, and the stimulus was surrounded by a gray border of $5^{\prime}$ that had the same mean luminance as the rest of the display.

The stimuli were made from the superposition of a spatiotemporally sinusoidal color grating and a spatiotemporally sinusoidal luminance grating both at the same spatial and temporal frequencies and $90^{\circ}$ out of phase with each other in both space and time [Eqs. (1) and (2)]. Three different color pairs were used, red/green, red/blue, and blue/green; each color was produced by one of the three phosphors of the television monitor. Each of the individual sine waves (red, green, or blue) for the color grating was at $100 \%$ contrast and added together in pairs $180^{\circ}$ out of phase. The luminance grating was produced by adding two sine waves of the same colors used to make the color grating and using the same mean luminance but with each wave now at $10 \%$ contrast and the two added together in phase. The spatial frequencies used were $0.5,1,2,4,8$, and $12 \mathrm{cpd}$. The temporal frequencies were $0.5,2$, and $7.5 \mathrm{~Hz}$.

For the blue/red stimuli, a minimum flicker condition at $7.5 \mathrm{~Hz}$ was run as well. The flicker stimulus is identical to the minimum-motion stimulus except that the luminance grating amplitude was zero $(m=0 \%)$, resulting in a simple counterphase flicker of the color grating [Eqs. (1) and (2)].

All 18 possible combinations of the six spatial and three temporal frequencies for a given color pair were run in random order with at least four readings for each condition. At the beginning of each trial, the stimulus was presented with a random relative luminance of the two colors being tested. The observer then adjusted a joystick to find the point at which the motion reversed direction. If there was a range within which no motion was seen, the observer tried to find 
an adjustment in the center of that range. Between trials, a spatially uniform field was displayed with the same mean luminance and mean chromaticity as the tests.

The maximum luminance of the red sine wave was set to $15 \mathrm{~cd} / \mathrm{m}^{2}$ as a reference, and the observers adjusted the luminances of the green and blue sine waves to find the equiluminance point. For the blue/green grating, green was taken as the fixed reference, and the luminance of the blue sine wave was varied. The fixed green luminance was set to the value required to match the $15 \mathrm{~cd} / \mathrm{m}^{2}$ of red at $0.5 \mathrm{~Hz}$ and 1 cpd for each observer.

As a check of the spatial and chromatic properties of the five-element achromatizing lens, the blue/green minimummotion condition was also run at $7.5 \mathrm{~Hz}$ and 2,4 , and $8 \mathrm{cpd}$ with a standard two-element achromatizing lens. The green and blue stimuli were appropriately scaled to compensate for the difference in magnification for these two colors introduced by this type of lens. The readings in this control condition were in good agreement with those obtained with the five-element lens.

We also verified the response of the monitor itself by measuring the luminance of individual black and colored bars of a square-wave grating as a function of its spatial frequency. The luminance contrast of the light and dark bars dropped slightly from the 0.5-cpd grating to the 8-cpd grating, the drop being smallest for a green grating $(0.3 \%)$ and slightly larger for red and blue gratings (2.9 and $3.1 \%$, respectively).

Finally, misregistration of the color images on the monitor will affect the strength of the motion signal produced by a luminance mismatch between the colors but not the equiluminance point, the point at which there is no motion [the components in Eqs. (1)-(3) are phase shifted by various amounts, introducing more flicker, but motion is still eliminated only if the two colors have the same luminance]. We verified this by introducing a misregistration between the two phosphor images on the screen of $45^{\circ}$ of phase in the 8-cpd test and found that this did not influence the settings. This misregistration is equivalent to $2.1 \mathrm{~mm}$ on the screen of the monitor, whereas the maximum misregistration of the red, green, and blue images when properly adjusted on the monitor is less than $1 \mathrm{~mm}$.

Two of the authors, SMA and PC, served as observers. Both have normal color vision and normal or corrected-tonormal acuity. SMA was unable to make settings at 8 or 12 cpd.

\section{Results}

The data are shown in Figs. 4-9. Figures 4 and 5 show that for red versus green there is little or no effect of spatial frequency on the equiluminance ratio, whereas there is a consistent effect of temporal frequency. The amount of green required to equal the luminance of the red increases by $9 \%$ for SMA (from 0.5 to $7.5 \mathrm{~Hz}$ ) and by $7 \%$ for PC. These results are consistent with those reported by Kelly ${ }^{19}$ in a threshold task.

Figures 6 and 7 show that there is a definite decrease in the contribution of the blue component compared with that of the red as spatial frequency increases. Significantly more blue (20 to $30 \%$ ) is required at higher spatial frequencies to match the constant $15-\mathrm{cd} / \mathrm{m}^{2}$ red component. There is, as well, an increase in the amount of blue required to match red

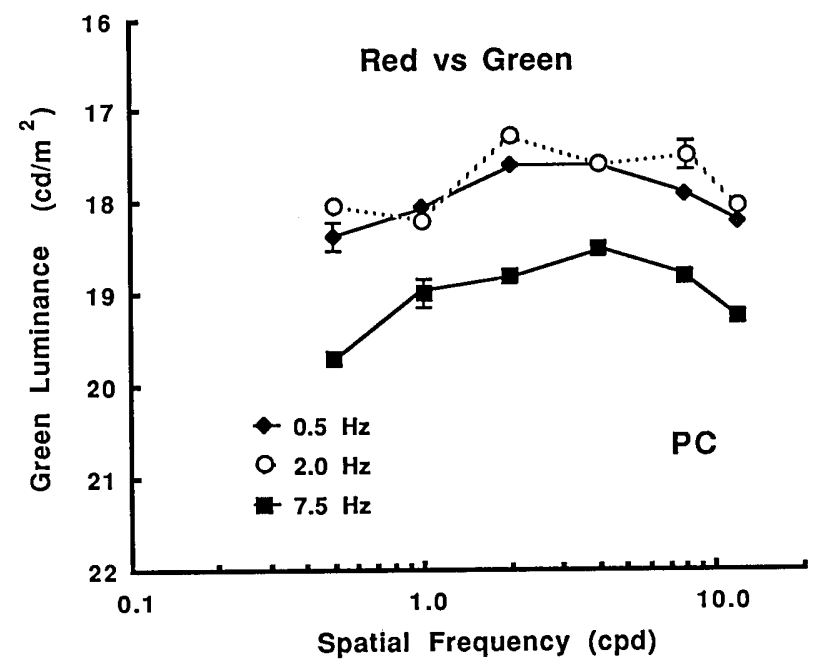

Fig. 4. The amplitude of the green component required to null the stimulus motion when combined with a $15-\mathrm{cd} / \mathrm{m}^{2} \mathrm{red}$ component, as a function of spatial and temporal frequency for observer PC. The vertical bars show the typical $( \pm 1)$ standard error. Note that to preserve the direction of a dropoff in blue efficiency with spatial frequency in later graphs, the luminance axis has been inverted here and in all following graphs except Fig. 9.

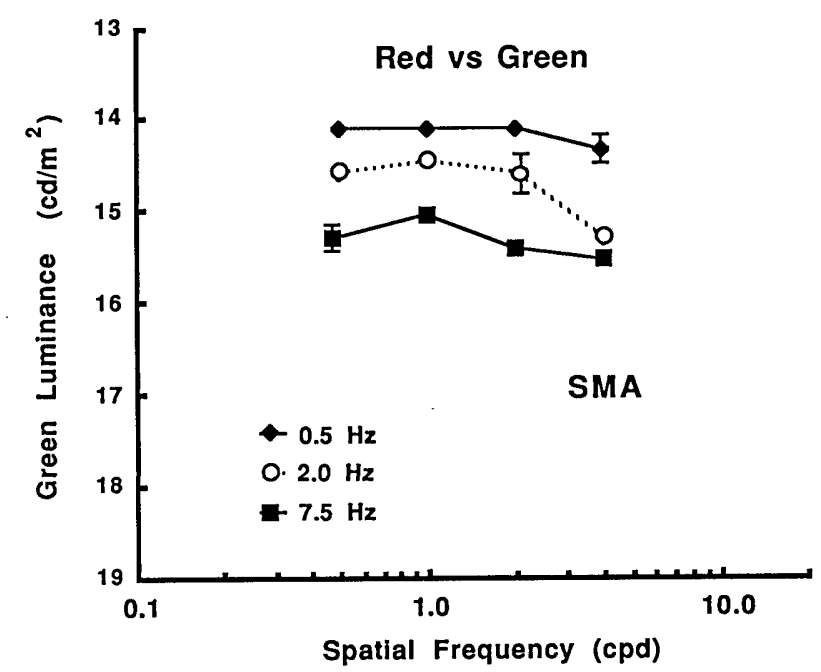

Fig. 5. The amplitude of the green component required to null the stimulus motion when combined with a $15-\mathrm{cd} / \mathrm{m}^{2}$ red component, as a function of spatial and temporal frequency for observer SMA. The vertical bars show the typical $( \pm 1)$ standard error.

as the temporal frequency increases that is similar to the increase seen in Figs. 4 and 5. Figures 6 and 7 also show the results for $7.5-\mathrm{Hz}$ minimum-flicker settings. These were in substantial agreement with the $7.5-\mathrm{Hz}$ minimum-motion settings.

Finally, Figs. 8 and 9 show that more blue is required to match green as the spatial frequency increases. There does not appear to be any effect of temporal frequency on the settings for SMA. For PC, however, the $0.5-\mathrm{Hz}$ condition requires more blue to match the green than do the 2- and 7.5$\mathrm{Hz}$ conditions. A similar small difference for $0.5 \mathrm{~Hz}$ with this observer also appears, but in the opposite direction, for the blue-versus-red data of Fig. 6. With the exception of this special case, then, temporal frequency does not appear to have an effect on the blue-versus-green settings. 
Since measurements were taken on all three combinations of red, green, and blue and since the measurements give an indication of the relative contributions of each color to the luminance channel, we should, in fact, be able to predict the third measurement, blue versus green, for example, from the other two, blue versus red and red versus green. If this prediction holds, we have found transitivity among the three measurements. Table 1 shows that the three sets of readings show very consistent transitivity for both observers.

Finally, to summarize the results of this experiment, temporal-frequency effects are evident when red is present in the stimulus (red contributes less to luminance at lower temporal frequencies), and spatial-frequency effects are found when blue is present (blue contributes less at higher spatial frequencies).

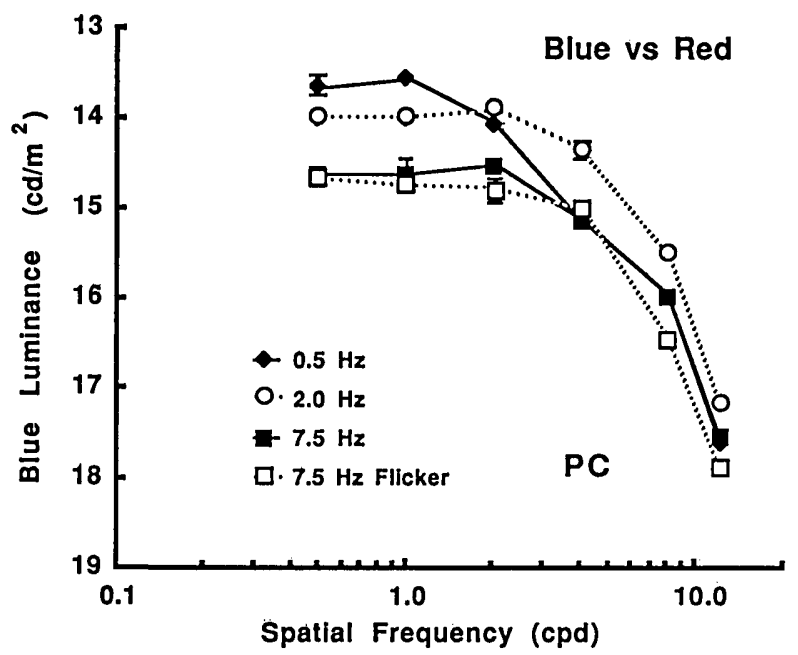

Fig. 6. The amplitude of the blue component required to null the stimulus motion when combined with a $15-\mathrm{cd} / \mathrm{m}^{2}$ red component, as a function of spatial and temporal frequency for observer PC. Data for minimum flicker settings at $7.5 \mathrm{~Hz}$ are also shown. The vertical bars show the typical $( \pm 1)$ standard error.

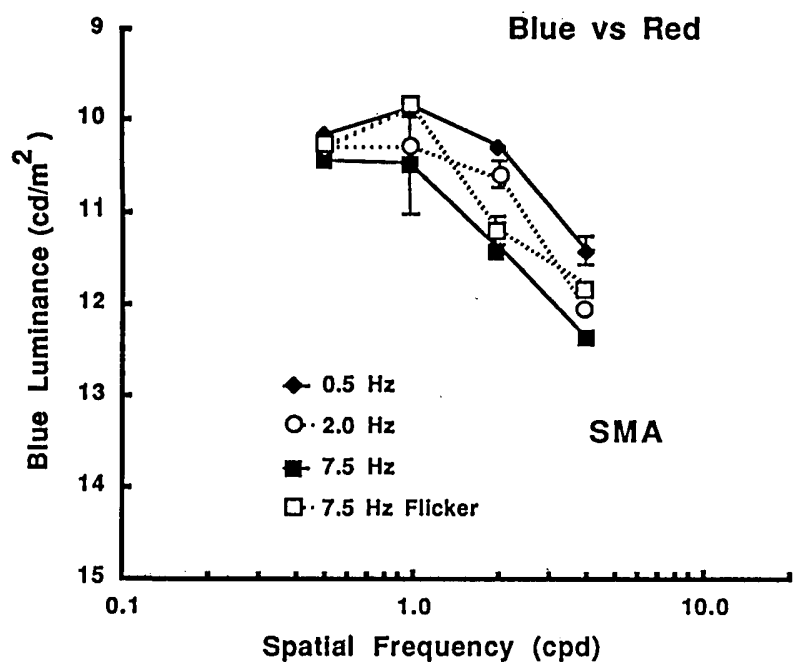

Fig. 7. The amplitude of the blue component required to null the stimulus motion when combined with a $15-\mathrm{cd} / \mathrm{m}^{2}$ red component, as a function of spatial and temporal frequency for observer SMA. Data for minimum flicker settings at $7.5 \mathrm{~Hz}$ aro also shown. Tho vertical bars show the typical $( \pm 1)$ standard error.

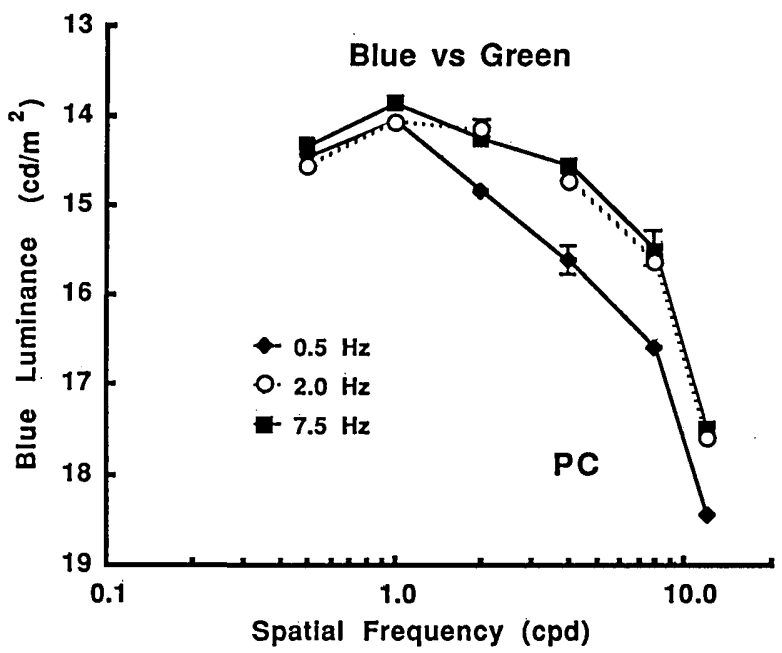

Fig. 8. The amplitude of the blue component required to null the stimulus motion when combined with an $18-\mathrm{cd} / \mathrm{m}^{2}$ green component, as a function of spatial and temporal frequency for observer PC. The vertical bars show the typical $( \pm 1)$ standard error.

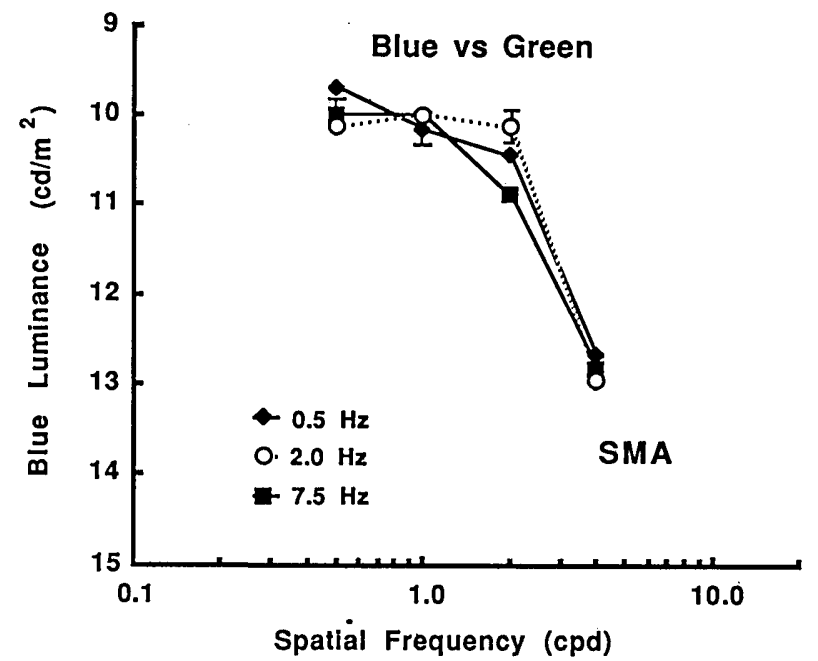

Fig. 9. The amplitude of the blue component required to null the stimulus motion when combined with a $14-\mathrm{cd} / \mathrm{m}^{2}$ green component, as a function of spatial and temporal frequency for observer PC. The vertical bars show the typical $( \pm 1)$ standard error.

Table 1. Transitivity of the Equiluminance Measures in Experiment 1 for Observers PC and SMA ${ }^{a}$

\begin{tabular}{ccccccccc}
\hline & $\begin{array}{c}\text { Temporal } \\
\text { Frequency }\end{array}$ & \multicolumn{6}{c}{$\begin{array}{c}\text { Transitivity of Equiluminance for the } \\
\text { Observer }\end{array}$} & \multicolumn{6}{c}{$\begin{array}{c}\text { Fowing Spatial Frequency (cpd) } \\
\text { (Hz) }\end{array}$} & 0.5 & 1 & 2 & 4 & 8 & 12 \\
\hline \multirow{2}{*}{ PC } & 0.5 & 0.93 & 0.96 & 1.04 & 0.99 & 0.97 & 0.95 \\
& 2.0 & 0.97 & 1.00 & 1.02 & 1.05 & 1.03 & 0.98 \\
& 7.5 & 0.93 & 1.00 & 0.98 & 1.01 & 0.99 & 1.03 \\
& & & & & & & \\
SMA & 0.5 & 1.04 & 0.96 & 0.98 & 0.89 & & \\
& 2.0 & 0.97 & 0.99 & 1.00 & 0.90 & & \\
& 7.5 & 0.93 & 0.97 & 0.94 & 0.87 & & \\
\hline
\end{tabular}

a The values shown are the product of the ratios of matching luminance values for green when matched with red and for blue when matched with green, divided by that for blue when matched with red. The expected value is 1.00; the average value for $\mathrm{PC}$ was 0.99 , and the average value for SMA was 0.95 . 


\section{EXPERIMENT 2. EQUILUMINANCE AFTER BLEACHING OF B CONES}

\section{Procedure}

The spatial-frequency effect seen when blue is involved but not when only red and green are involved might reflect the sparser sampling of the retinal image by the $\mathrm{B}$ cones ${ }^{20}$; that is, the response of the blue mechanisms will drop as the spatial frequency of the blue variation in the stimulus approaches the sampling density of the $B$ cones. This interpretation requires us to assume, despite evidence to the contrary, ${ }^{8}$ that $\mathrm{B}$ cones contribute to luminance. On the other hand, the spatial-frequency effect for blue stimuli may result from inhomogeneities within the macular pigment. ${ }^{21}$ Higher spatial frequencies are better detected by more central parts of the retina, and the yellow macular pigmentation filters out more and more blue at smaller eccentricities. In order to test these possibilities, we next bleached the $B$ cones to see whether the settings would be affected when the B cones no longer contributed any response. To test for inhomogeneities within and outside the macula we used three field sizes: $1^{\circ}, 2^{\circ}$, and $12^{\circ}$. For the $12^{\circ}$ field, the fixation bull's-eye was expanded to cover the central $4^{\circ}$ in order to exclude the macular area.

Using the minimum-motion technique described in experiment 1 , we determined equiluminance settings, always at $2 \mathrm{~Hz}$, for green versus blue at several spatial frequencies: $1,2,4$, and $8 \mathrm{cpd}$ for the $1^{\circ}$ display; $0.5,1,2,4$, and $8 \mathrm{cpd}$ for the $2^{\circ}$ display; and $0.25,0.5,1,2$, and $4 \mathrm{cpd}$ for the $12^{\circ}$ display. For the $2^{\circ}$ and $12^{\circ}$ displays, these conditions were run in random order with the $\mathrm{B}$ cones bleached and, in a separate session, without bleaching. For the $1^{\circ}$ field, the measurements were made only without bleaching.

In order to bleach the B cones, each observer exposed his eye to be tested to approximately $4800 \mathrm{Td}$ of violet light for 1 min. The light was produced by focusing the beam of a 300-W Kodak Carousel with a reflector-type lamp through a reversed $f / 2.8,35-\mathrm{mm}$ lens and filtering it through a $435-\mathrm{nm}$ interference filter having a 7 -nm half-bandwidth at halfamplitude. The resulting beam was viewed through a natural pupil measured at approximately $3 \mathrm{~mm}$. The CIE $x$ and $y$ coordinates of the light, measured with a Minolta chromatometer, were 0.16 and 0.01 . The effectiveness of the bleach was evaluated by determining that a grating made of two colors along each observer's tritanopic confusion line appeared achromatic and by observing the time course of the return of chromatic sensations under conditions chosen to optimize chromatic sensitivity [2 Hz, 2 cpd (Ref. 19)]. The bleach produced the effect that no chrominance could be seen in the tritanopic stimulus for approximately $1 \mathrm{~min}$.

For the experimental conditions, the observer first exposed himself to the bleaching light for $1 \mathrm{~min}$ and then moved immediately to the minimum-motion display and made settings for up to $1 \mathrm{~min}$ or until he observed a change of the chrominance in the display. Since the stimulus colors, blue and green, did not fall along the tritanopic confusion line, the stimulus was not achromatic after the bleach but appeared to be blue-green and gray, respectively. As the bleach wore off, a very noticeable yellow would fill the gray stripes, turning finally to green. The blue-green would simultaneously shift to saturated blue. If the observers no- ticed any chromatic changes before the minute had elapsed, they stopped making readings. At least four settings were made in each condition.

The observers were two of the authors, $\mathrm{PC}$ and $\mathrm{DM}$ (DM is a deuteranomalous trichromat with normal acuity). For the $12^{\circ}$ field, blue luminance was held at $10 \mathrm{~cd} / \mathrm{m}^{2}$, and green luminance was varied to find the equiluminance settings; for the $1^{\circ}$ and $2^{\circ}$ fields, blue was held at $16 \mathrm{~cd} / \mathrm{m}^{2}$ and green was varied for $\mathrm{PC}$, whereas green was fixed at $15 \mathrm{~cd} / \mathrm{m}^{2}$ and blue was varied for DM.

\section{Results}

The results for the $1^{\circ}$ and $2^{\circ}$ fields are shown in Figs. 10 and 11. The decrease in green in Fig. 10 (or, equivalently, the

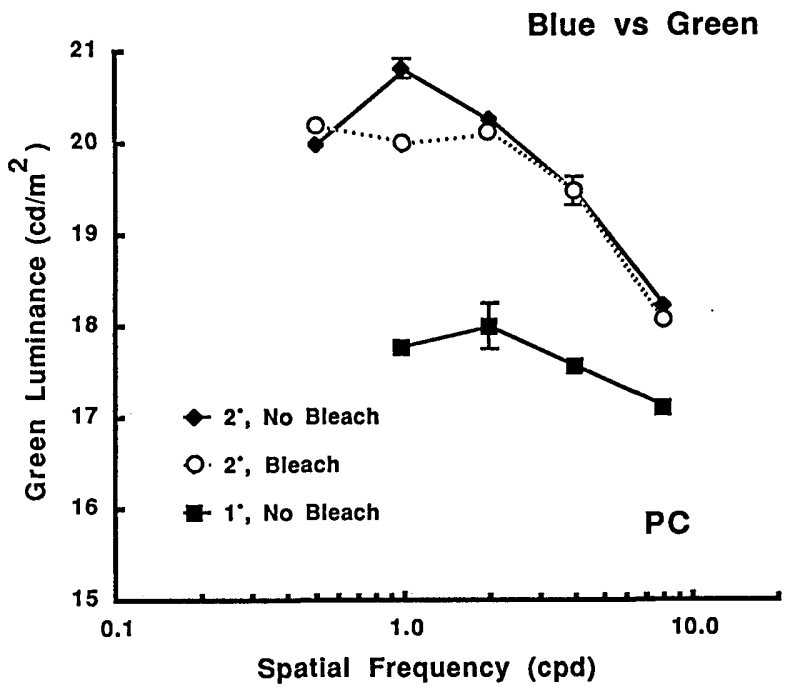

Fig. 10. The effect of bleaching the $B$ cones and of a smaller $\left(1^{\circ}\right)$ field on the amplitude of the green component required to null the stimulus motion when combined with a $16-\mathrm{cd} / \mathrm{m}^{2}$ blue component, as a function of spatial frequency for observer PC. Note that the luminance axis is not inverted in this graph. The vertical bars show the typical $( \pm 1)$ standard error.

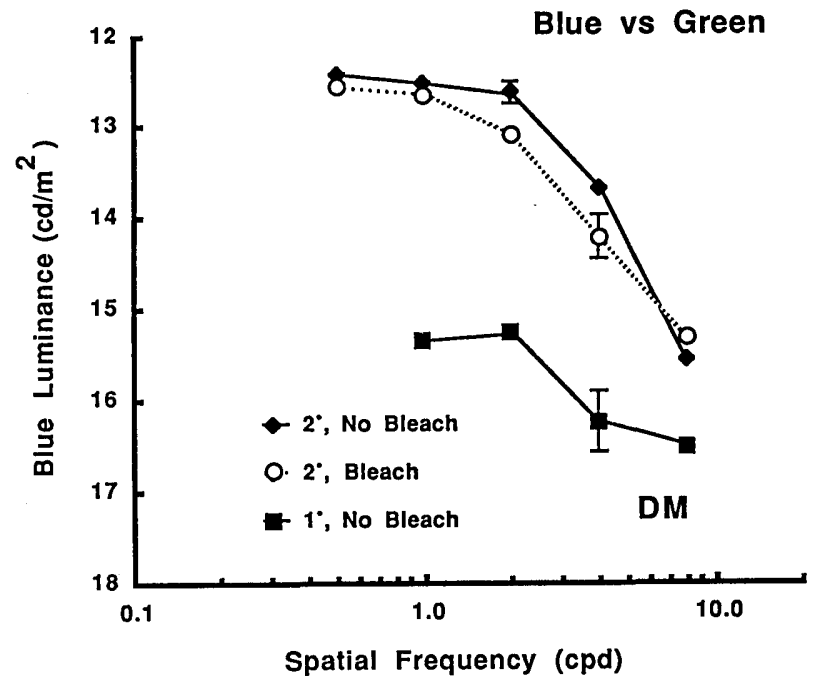

Fig. 11. The effect of bleaching the $B$ cones and of a smaller $\left(1^{\circ}\right)$ field on the amplitude of the blue component required to null the stimulus motion when combined with a $15-\mathrm{cd} / \mathrm{m}^{2}$ green component, as a function of spatial frequency for observer DM. The vertical bars show the typical $( \pm 1)$ standard error. 
increase in blue in Fig. 11) that is required for equiluminance to be reached as spatial frequency increases is found again here without bleaching but is also found after bleaching of the $\mathrm{B}$ cones. In fact, bleaching the $\mathrm{B}$ cones appears to have very little effect on the equiluminance settings at all, implying that these cones are not contributing to the luminance response for either the green or the blue stimulus.

On the other hand, changing the field size to $1^{\circ}$ substantially alters the equiluminance settings. The settings with the smaller field are close to those for the higher spatial frequencies in the $2^{\circ}$ field and vary much less with spatial frequency. This is as expected if the source of the spatialfrequency effect is an inhomogeneity in the macular pigmentation, with more blue being absorbed at smaller eccentricities. The central $1^{\circ}$ will then be less sensitive to blue.

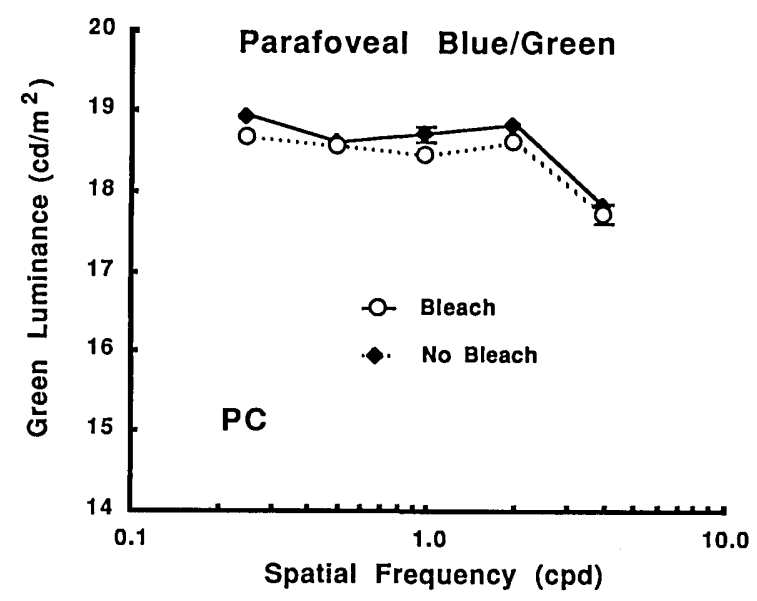

Fig. 12. The effect of bleaching the parafoveal $\mathrm{B}$ cones $\left(2^{\circ}\right.$ to $6^{\circ}$ eccentricity) on the amplitude of the green component required to null the stimulus motion when combined with a $10-\mathrm{cd} / \mathrm{m}^{2}$ blue component, as a function of spatial frequency for observer PC. Note that the luminance axis is not inverted in this graph. The vertical bars show the typical $( \pm 1)$ standard error.

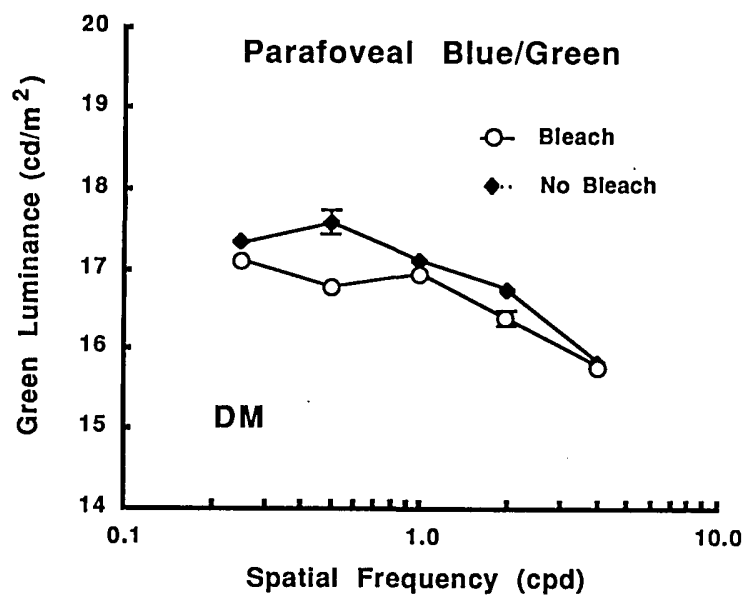

Fig. 13. The effect of bleaching the parafoveal $B$ cones $\left(2^{\circ}\right.$ to $6^{\circ}$ eccentricity) on the amplitude of the green component required to null the stimulus motion when combined with a $10-\mathrm{cd} / \mathrm{m}^{2}$ blue component, as a function of spatial frequency for observer DM. Note that the luminance axis is not inverted in this graph. The vertical bars show the typical $( \pm 1)$ standard error.
The settings in the $1^{\circ}$ field will be shifted toward those made for higher spatial frequencies in the larger field because both of these cases involve detection by the central fovea alone. The settings in the $1^{\circ}$ field change less with spatial frequency because the amount of inhomogeneity within $1^{\circ}$ is smaller both in pigmentation and in frequency response.

The results for the $12^{\circ}$ field are shown in Figs. 12 and 13. For this parafoveal region, $2^{\circ}$ to $5^{\circ}$ eccentricity, there is again little effect of bleaching on the equiluminance settings. There is also less effect of spatial frequency on the settings, as would be expected if this display fell outside the macular pigmentation and its inhomogeneities.

Although the effects of bleaching are small, they are fairly consistent across spatial frequency and field size. After bleaching, the green luminance required for equiluminance with blue decreased by an average of $1.0 \%$ (standard error of $\pm 0.4 \%$ ) for PC and $1.8 \%$ (standard error of $\pm 0.6 \%$ ) for DM.

\section{DISCUSSION}

Our results show that the $\mathrm{B}$ cones do not contribute appreciably to the luminance pathway under the conditions of these experiments. Our readings were taken at low spatial and temporal frequencies for both foveal and parafoveal regions and so respond to Drum's criticism ${ }^{13}$ of previous work. The effect of bleaching the $\mathrm{B}$ cones on the equiluminance settings for green versus blue was less than 1 or $2 \%$, and we claim that this is the upper limit of the possible contribution of the $\mathrm{B}$ cones to the luminance pathway. On the other hand, the effects of the bleaching light on selfscreening 22 in the $R$ and $G$ cones can be expected to produce a change of about 1 or $2 \%$ in the same direction as that observed, and so there may in fact be no contribution from the $\mathrm{B}$ cones. In either case, $\mathrm{B}$ cones have a minimal effect on the luminance pathway, and blue stimuli must affect the luminance pathway almost entirely through the $R$ and the $G$, or long- and medium-wavelength, cones.

Although the blue variation in the stimulus acts through the $\mathrm{R}$ and $\mathrm{G}$ cones, it is still susceptible to filtering by the macular pigment. We attribute the spatial-frequency effects on equiluminance matches involving blue stimuli to the inhomogeneity in the macular pigmentation. Since highspatial-frequency stimuli will be detected predominantly by the central fovea and lower spatial frequencies will be detected over a greater area, different spatial frequencies will sample different areas of the fovea and receive different amounts of filtering.

Although the spatial-frequency effect seen for blue stimuli appears not to be due to receptor factors, the temporalfrequency effect seen for red stimuli may be due to differences in temporal responses of the $G$ and $R$ cones. The effect that we report is similar to that found by Kelly ${ }^{19}$ for a threshold task. The temporal-frequency effect seen in both these studies suggests that the equality of heterochromatic. matches made by flicker techniques at fairly high temporal rates $^{23}$ ( 8 to $20 \mathrm{~Hz}$ ), and those made for static fields using minimally distinct border techniques may involve some additional factor. Based on our results here, we would predict a difference between the two techniques of about $5-10 \%$. Since this is not observed, an additional factor, perhaps adaptation, ${ }^{24}$ may be counteracting the difference caused by temporal frequency. 
The small spatial-frequency dependence of the luminance null in the red-green spectral range is interesting for another reason. The physiological substrate of the luminance-sensitive pathway is not clear. It is possible that it incorporates the chromatically opponent Wiesel and Hubel ${ }^{25}$ type 1 cells, which have centers fed by one cone type and surrounds fed by another; signals from red-center and green-center cells might be averaged to form a luminance signal. If this model were correct, any mismatch in scale between the receptive field profiles of the red-and green-center cells would cause the spectral sensitivity of the luminance channel to vary with spatial frequency. Thus, either this model is not correct or the receptive fields of the red- and green-center cells do not differ substantially.

The small effect of spatial frequency for the red/green matches that we report here, although also consistent with Kelly's findings, does not agree with a recent report by Mullen. ${ }^{26}$ She used a threshold task similar to Kelly's but found that the efficiency of luminance response to red increased at higher spatial frequencies compared with the response to green. This finding is similar to our first results with the minimum-motion technique using square-wave stimuli. ${ }^{14}$ We attribute our earlier finding of a spatial-frequency effect for red/green to chromatic aberration, which was not controlled in our previous study. Having corrected for chromatic aberration in this study, we conclude that there is little effect of spatial frequency on red/green matches.

The effects of spatial and temporal frequency that we have found appear to be attributable to optical (macular pigmentation) and receptor factors. The data do not provide any strong evidence requiring the assumption of several luminance pathways having different properties, nor is there any indication that the different techniques used to measure luminance might be evaluating different luminance subsystems. We obtained similar equiluminance settings for our minimum-motion technique and for minimum flicker, and other studies have shown similar equiluminance settings for flicker matches at high temporal frequencies and minimum border matches for stimuli with no temporal variation. ${ }^{23}$ We have also extended the evaluation of equiluminance settings to situations involving perception of static, achromatic forms such as shadow figure ${ }^{27}$ and subjective contours ${ }^{28}$ and still find no evidence of separate luminance pathways for these conditions. ${ }^{29}$

Our data also provide important guidelines for the construction of purely chromatic images. Such images provide no pattern for the luminance pathway and so are useful for studying the properties of the opponent-color pathways in isolation. First, blue should be avoided in constructing such equiluminous images because of the inhomogeneity of blue absorption across the retina, not only inside versus outside the $2^{\circ}$ macular area but within the macula as well, as we have shown here. It would be difficult to produce a uniformly equiluminous stimulus of more than $1^{\circ}$ in size when blue is involved, and, even in this case, fixation would have to be carefully maintained. The problem would be less serious if blue areas of the stimulus were completely outside the macular region, but, again, fixation would have to be carefully controlled to prevent the blue portions of the stimulus from falling within the macula.

Unlike images involving blue, those involving red and green would not suffer from spatial inhomogeneity. Howev- er, temporal factors would be a problem. Temporal frequencies should therefore be kept within a restricted range either by fixating static targets or by making target appearances and disappearances occur gradually.

Although the effects of spatial and temporal frequency appeared to be fairly consistent from observer to observer in our experiments, there were substantial differences in mean equiluminance settings between individuals. For example, in experiment $1, \mathrm{PC}$ required 30 to $40 \%$ more green or blue luminance to match the red standard than did SMA. This wide variation between color normals is not unusual ${ }^{30}$ and underlines the importance of individual adjustments in any task requiring equiluminous colors.

Finally, chromatic aberration always produces problems whenever high spatial frequencies are involved, as the observer can control the relative contrast of the stimulus colors by changing accommodation to bring the different color independently into focus. The contrast of the out-of-focus image colors is reduced by blurring, and this contrast loss is greater the finer the detail (the higher the spatial frequency content) of the image. Therefore either images should be blurred to reduce the high spatial-frequency content or the observers must be corrected for chromatic aberration. If a two-element lens is used for correcting the axial aberration, then the relative scales of the different-colored images must be adjusted to compensate for the differential magnification introduced by these lenses. Alternatively, a lens that does not introduce differential magnification ${ }^{16,31,32}$ can be used.

\section{ACKNOWLEDGMENTS}

This research was supported in part by grants A8606 and A0260 from the Natural Science and Engineering Research Council of Canada to P. Cavanagh and S. M. Anstis. We are grateful to Charles Stromeyer for his comments on an earlier draft of this paper.

\section{REFERENCES}

1. P. Gouras, "Identification of cone mechanisms in monkey ganglion cells," J. Physiol. 199, 533-547 (1968).

2. F. M. de Monasterio and P. Gouras, "Functional properties of the rhesus monkey retina," J. Physiol. 251, 167-195 (1975).

3. F. M. de Monasterio, "Properties of concentrically organized X and $Y$ ganglion cells of macaque retina," J. Neurophysiol. 41, 1394-1417 (1978).

4. E. Zrenner and P. Gouras, "Characteristics of the blue-sensitive cone mechanisms in primate retinal ganglion cells," Vision Res. 21, 1605-1609 (1981).

5. E. Zrenner, Neurophysiological Aspects of Color Vision in Primates (Springer-Verlag, Berlin, 1983).

6. P. H. Schiller and C. L. Colby, "The responses of single cells in the lateral geniculate nucleus of the rhesus monkey to color and luminance contrast," Vision Res. 23, 1631-1641 (1983).

7. C. R. Ingling and E. Martinez-Uriegas, "The spatiotemporal properties of the r-g X-cell channel," Vision Res. 25, 33-38 (1985).

8. A. Eisner and D. I. A. MacLeod, "Blue-sensitive cones do not contribute to luminance,” J. Opt. Soc. Am. 70, 121-123 (1980).

9. B. W. Tansley and R. M. Boynton, "Chromatic border preception: the role of red- and green-sensitive cones,"Vision Res. 18, 683-697 (1978).

10. G. S. Brindley, J. J. DuCroz, and W. A. H. Rushton, "The flicker fusion frequency of the blue sensitive mechanism of colour vision," J. Physiol. 183, 497-500 (1966).

11. D. H. Kelly, "Spatio-temporal frequency characteristics of color-vision mechanisms," J. Opt. Soc. Am. 64, 983-990 (1974). 
12. J. J. Wisowaty and R. M. Boynton, "Temporal modulation sensitivity of the blue mechanism: measurements made without chromatic adaptation," Vision Res. 20, 895-909 (1980).

13. B. Drum, "Short-wavelength cones contribute to achromatic sensitivity," Vision Res. 23, 1433-1439 (1983).

14. S. M. Anstis and P. Cavanagh, "A minimum motion technique for judging equiluminance," in Colour Vision: Physiology and Psychophysics, J. D. Mollon and L. T. Sharpe, eds. (Academic, London, 1983), pp. 156-166.

15. P. Cavanagh, S. Anstis, and G. Mather, "Screening for color blindness using optokinetic nystagmus," Invest. Ophthalmol. Vis. Sci. 25, 463-466 (1984).

16. E. Levinson and R. Sekuler, "The independence of channels in human vision selective for direction of movement," J. Physiol. 250, 347-366 (1975).

17. C. F. Stromeyer III, R. E. Kronauer, J. C. Madsen, and S. A. Klein, "Opponent-movement mechanisms in human vision," J. Opt. Soc. Am. A 1, 876-884 (1984).

18. I. Powell, "Lenses for correcting chromatic aberration of the eye," Appl. Opt. 20, 4155-4157 (1981).

19. D. H. Kelly, "Spatiotemporal variation of chromatic and achromatic contrast thresholds," J. Opt. Soc. Am. 73, 742-750 (1983).

20. D. R. Williams and R. J. Collier, "Consequences of spatial sampling by a human photoreceptor mosiac," Science 221, 385-387 (1983).

21. D. R. Williams, D. I. A. MacLeod, and M. Hayhoe, "Punctate sensitivity of the blue-sensitive mechanism," Vision Res. 21, 1357-1375 (1981).
22. W. S. Stiles and G. Wyszecki, "Colour-matching data and the spectral absorption curves of visual pigments," Vision Res. 14, 195-207 (1974).

23. G. Wagner and R. M. Boynton, "A comparison of four methods of heterochromatic photometry," J. Opt. Soc. Am. 62, 15081515 (1972).

24. G. A. Fry, "Adaptation and heterochromatic brightness matching," Invest. Ophthalmol. Vis. Sci. Suppl. 21, 134 (1980).

25. T. N. Wiesel and D. H. Hubel, "Spatial and chromatic interactions in the lateral geniculate body of the rhesus monkey," J. Neurophysiol. 29, 1115-1156 (1966).

26. K. T. Mullen, "The contrast sensitivity of human color vision to red-green and blue-yellow chromatic gratings," J. Physiol. 359, 381-400 (1985).

27. P. Cavanagh and Y. Leclerc, "Shadow constraints," Invest. Ophthalmol. Vis. Sci. Suppl. 26, 282 (1985).

28. P. Cavanagh, "Subjective contours signalled by luminance, vetoed by motion or depth," Bull. Psychon. Soc. 23, 273 (1985).

29. P. Cavanagh, S. Shioiri and D. I. A. MacLeod, "Is the achromatic form pathway based on brightness or luminance?" Invest. Ophthalmol. Vis. Sci. Suppl. 28, 362 (1987).

30. R. A. Crone, "Spectral sensitivity in color-defective subjects and heterozygous carriers," Am. J. Ophthalmol. 48, 231-235 (1959).

31. A. L. Lewis, M. Katz and C. Oehrlein, "A modified achromatizing lens," Am. J. Optom. Physiol. Opt. 59, 909-911 (1982).

32. P. A. Howarth and A. Bradley, "The longitudinal chromatic aberration of the human eye and its correction," Vision Res. 26, 361-366 (1986). 